\title{
Um Estágio em Medicina da Família e Comunidade no País Irmão
}

\author{
An Internship in The Brother Country - Practice Report of an Internship \\ In Family Medicine In Brazil
}

Joana Rita Marinho

\section{Resumo}

Introdução: Atualmente, vivemos num mundo globalizado. A migração populacional é cada vez maior, com predomínio do fluxo de pessoas de países desfavorecidos, para países com maior estabilidade socioeconómica. Nos últimos anos, a população migrante oriunda do Brasil tem aumentado em Portugal, particularmente em Lisboa. Na minha prática diária, tenho-me deparado frequentemente com emigrantes brasileiros, com as suas particularidades clínicas, crenças e cultura, que têm impacto na sua saúde individual. Assim, senti necessidade de conhecer melhor a realidade do Brasil, em termos dos cuidados de saúde e da sua cultura. Por outro lado, tinha como objetivo o conhecimento no terreno da forma de organização dos Cuidados de Saúde Primários de outro país - experiência enriquecedora para a prática clínica de um futuro médico de família.

Objetivo(s) e Métodos: Realizar um estágio num centro de saúde do Rio de Janeiro (Brasil) em setembro de 2018, orientado por um médico de medicina da família e da comunidade, para conhecer melhor a realidade brasileira, em termos culturais, a organização dos cuidados de saúde, e melhorar a minha prática clínica futura.

Resultados: 0 estágio possibilitou conhecer aprofundadamente o sistema de saúde brasileiro, em particular os cuidados de saúde básicos do RJ, assim como aspetos da cultura, organização e funcionamento social da população.

\begin{abstract}
Introduction: Nowadays, we live in a globalized world. Population migration is increasing, predominantly with a flow of people from deprived countries to countries with greater socioeconomic stability. In the past years, the migrant population coming from Brazil has increased in Portugal, particularly in Lisbon. As a family doctor, I have often encountered Brazilian emigrants, with their clinical peculiarities, beliefs and culture, which have an impact on individual health. Thus, I felt the need to become better acquainted with the reality of Brazil, in terms of health care and its culture. On the other hand, another goal was to get to know, in the field, another Primary Care form of organization - an enriching experience for any future family doctor.
\end{abstract}

Objective(s) and Methods: To carry out an internship in a health center in Rio de Janeiro (Brasil) at September 18, guided by a family and community doctor, to better understand the Brazilian culture and health care system.

Results: It was possible to get to know in depth the Brazilian health care system, in particular the primary health care of RJ, as well as the culture, organization and social functioning of the population.

Conclusion: It was an enriching internship, which made it possible to know a different health care system, innovative, that surpassed my expectations. It also made possible the training and increase of the medical knowledge, mainly of less frequent pathologies in Portugal. 
Conclusão: Foi um estágio muito enriquecedor em termos profissionais, tendo possibilitado conhecer um sistema de saúde em cuidados básicos diferente, inovador, que superou as minhas expectativas. Também possibilitou o treino e aumento do conhecimento médico, principalmente de patologias menos frequentes em Portugal. Por outro lado, houve um crescimento pessoal com a integração temporária na comunidade do Rio de Janeiro e vivência sociocultural. Em última análise, permitiu-me conhecer melhor uma população migrante e adequar os cuidados de saúde e abordagem médica a esta população crescente no meu país.

Palavras-chave: medicina geral e familiar, Brasil, educação médica

\section{Introdução}

Em 1978, foi promovida conjuntamente pela Organização Mundial da Saúde (OMS) e pela UNICEF, a “Declaração de Alma-Ata" ${ }^{1}$, que veio definir os Cuidados de Saúde Primários (CSP) como "os cuidados essenciais de saúde" que "são levados o mais proximamente possível aos lugares onde pessoas vivem e trabalham, e constituem o primeiro elemento de um processo contínuo de assistência em saúde." Os CSP são o pilar dos sistemas de saúde em inúmeros países a nível mundial. As fronteiras entre os vários países têm vindo a esbater-se, no sentido de que cada vez mais somos cidadãos do mundo. Estima-se que, durante o ano de 2017, tenham entrado em Portugal 19551 imigrantes permanentes oriundos de cinco principais países: Brasil (15\%), França (12\%), Reino Unido (11\%), Suiça (11\%) e Venezuela $(6 \%)^{2}$. Relativamente à população estrangeira com estatuto de residente, em 2017, eram 416682 e, desde 2014, que o posicionamento das cinco principais nacionalidades se mantém inalterado: Brasil, Cabo Verde, Ucrânia, Roménia e China. ${ }^{2}$

A população migratória brasileira é muito significativa em Portugal, com um total de 85.426 cidadãos $^{3}$, mantendo-se como a principal comunidade estrangeira residente. Destes, a maioria está alocada à região de Lisboa e Vale do Tejo. Pelos dados do INE ${ }^{4}$, residem 40 385 na área metropolitana de Lisboa, sendo que existem 2469 brasileiros no concelho de Almada e 1629 no concelho do Seixal, o que significa que aproximadamente $1 / 3$ da população brasileira está localizada na área do ACeS Almada-Seixal.
On the other hand, there was a personal growth with the temporary integration in the community of RJ and consequent sociocultural experience. Ultimately, it allowed me to better understand a migrant population and to tailor health care and medical approach to this growing population in my country.

Keywords: general practice, Brazil, medical education

A realidade descrita acima foi um dos fatores que me motivou a selecionar o Brasil como local para estágio em cuidados de saúde primários no estrangeiro.

A curiosidade em conhecer outras realidades, perceber e integrar outras culturas, outras perspetivas e outras organizações de cuidados de saúde foi algo sempre presente desde que integrei o internato em MGF. Pareceu-me que o estágio de MGF3 seria o momento oportuno, tendo em conta que seria uma altura em que já teria um bom conhecimento e alguma prática do modo de funcionamento dos CSP. Vi este estágio como uma oportunidade para contactar com uma realidade médica, populacional e cultural diferente, tendo em vista o meu desenvolvimento profissional e pessoal. Também me pareceu oportuno para potenciar o meu conhecimento médico, através da experiência de contacto com uma população diferente e heterogénea, que pudesse vir a ser aplicado na minha prática futura em Portugal, nomeadamente ao nível de algumas doenças mais prevalentes no Brasil, tendo em conta o fluxo de migrantes para o nosso país, que nos traz as suas peculiaridades culturais e de saúde.

Por outro lado, pareceu-me uma mais valia conhecer um sistema de cuidados de saúde diferente, e perceber a sua história, a sua organização e o seu modo de funcionamento, assim como perceber as semelhanças e diferenças face ao nosso SNS e, eventualmente, servir como inspiração para algumas melhorias da prática clínica. 
Em última análise, achei de extrema importância realizar este desafio para o meu próprio desenvolvimento pessoal e, consequentemente, profissional - uma oportunidade única de crescer, aprender e evoluir.

\section{Métodos}

Pelos motivos descritos, propus-me a um estágio curto durante o período de estágio de MGF3, com a duração de 80 horas, no Rio de Janeiro (RJ), numa clínica de família, tutoreada por um médico especialista em Medicina da Família e da Comunidade (MFC). Foi uma oportunidade única de crescer, aprender e evoluir como pessoa e como futura médica de família. Nas próximas secções irei relatar a minha experiência, abordando a organização dos cuidados de saúde brasileiros, particularmente do estado do RJ, assim como o quão enriquecedor foi este estágio, que decorreu entre 3 e 14 de setembro de 2018.

\section{Resultados}

No Brasil, a Atenção Primária à Saúde (APS) é vista como o primeiro nível de acesso ao um sistema de saúde - o Serviço de Saúde Único (SUS). ${ }^{5}$ Atualmente, a principal estratégia de políticas de saúde no Brasil é a saúde da família que tem recebido importantes incentivos financeiros, visando à ampliação da cobertura populacional e à reorganização da atenção. A saúde da família aprofunda os processos de territorialização e responsabilidade sanitária das equipas de saúde, compostas basicamente por médico(s) generalista(s), enfermeiro, auxiliares de enfermagem e agentes comunitários de saúde, cujo trabalho é referência de cuidados para a população. ${ }^{6}$ No RJ, o desenvolvimento da atenção primária tem sido particularmente notório e admirável. Irei relatar, conjuntamente, com a minha experiência, a organização do SUS no RJ.

No primeiro dia, fui recebida na Coordenação da PRMFC, onde foi feita uma apresentação breve do SUS, nomeadamente sobre a organização da APS. É exemplar a organização e estrutura que adquiriram em menos de 10 anos. Tem sido um enorme esforço aliado a um espírito de inovação e desenvolvimento que têm caracterizado a formação médica e os cuidados prestados à população neste período. O RJ passou de ter apenas 3\% da comunidade abrangida por um médico de família para atualmente terem cerca de $70 \%$ da população coberta. A residência em MFC tem a duração de dois anos. No primeiro, a formação ocorre na própria unidade de saúde (Centro Municipal de Saúde ou nas Clínicas da Família), junto do preceptor (orientador de formação). É suposto neste ano terem autonomia suficiente e conseguirem gerir os problemas mais comuns e o tempo de consulta, bem como executar os mais diversos procedimentos e prescrições. O segundo ano de internato está mais vocacionado para estágios no exterior (hospital ou outros serviços de saúde), sendo que apenas uma pequena parte do tempo é passada na unidade.

\section{A Residência no RJ}

Saliento algumas coisas que considerei interessantes na formação dos internos. Existe um Programa Político Pedagógico que determina um período de 6 horas semanais, distribuídas por várias atividades devidamente calendarizadas: sessões clinicas ( $2 \mathrm{~h} / \mathrm{semana}$ ), discussão de temas clínicos divididos em 5 eixos temáticos (cardiovascular, materno-infantil, saúde mental e indiferenciados I e II), seminários e/ou oficinas de capacitação a cada 3 meses (espaços multimédia com videoconferência - 12h), oficinas semanais com capacitação de preceptores e matriciamento (consultoria) com preceptores de outras especialidades. Do que presenciei na clínica onde estive, existe uma reunião semanal, com internos e, por vezes, com a participação de um ou mais preceptores, onde se discutem casos clínicos, onde se fazem apresentações teóricas/teórico-práticas dos mais variados temas, onde se discutem videogravações, onde se fazem outro tipo de apresentações e onde se esclarecem e discutem questões relacionadas com a residência. Curiosamente, no primeiro dia, pude assistir à exposição de uma das residentes sobre um estágio que tinha feito recentemente em Portugal na USF Oriente, sendo que considerei muito interessante, pois foi comparando o nosso sistema (que conseguiu conhecer com bastante detalhe) com o sistema brasileiro, o que me deu também logo uma perspetiva. Existem também aulas que são lecionadas pelos preceptores aos seus internos ou a um grupo de internos, baseadas nuns guiões desenvolvidos pelo grupo de coordenação do internato, que consistem na discussão de vários temas baseada em casos clínicos dos cuidados de saúde primários.

Não existe um sistema de avaliação geral para os internos - há avaliações trimestrais com os preceptores e, no final dos dois anos, é o preceptor que determina se o residente está apto para ser especialista em MFC ou se necessita de prolongar o tempo de residência. A título de curiosidade, um residente de MFC no RJ, na altura do estágio, tinha um salário mensal de cerca de 3000 reais (aproximadamente 680€), que é praticamente o dobro do que aufere um residente hospitalar. Um especialista de MFC, tem um rendimento mensal à volta de 7500 reais (aproximadamente $1700 €$ ). 
Em dois anos, os internos têm de adquirir uma quantidade de conhecimento e competência muito considerável, pelo que considero que a estrutura atual da residência está muito bem desenhada para que tal seja possível. Claro que isso acarreta um esforço significativo da parte dos residentes, que têm horários semanais acima das 50 horas, fora tempo de estudo e trabalhos. Contudo, têm também uma tarde sem consulta presencial, onde fazem outro tipo de atividades, nomeadamente atualizar o processo de alguns utentes, no que respeita a vigilâncias, pedidos de encaminhamento (referenciações hospitalares), entre outros. Fiquei com a impressão que desde o primeiro ano, os internos são muito autónomos e gerem eles próprios os doentes que vão atendendo, sendo que agem mais como médicos de família destas pessoas, dos que os próprios preceptores, pois a maior parte destes últimos exerce fundamentalmente um papel de consultoria.

\section{o Sistema Único de Saúde - Clínicas da Família}

Cada médico de família tem entre 3000 a 4000 utentes atribuídos. É extremamente difícil gerir uma lista com esta dimensão. Ainda para mais com a viragem económica dos últimos anos, muitas pessoas perderam a capacidade de poder pagar planos de saúde (equivalente aos seguros de saúde/subsistemas de saúde em Portugal). Assim, muitos ficaram unicamente dependentes do SUS, que ainda não tem capacidade suficiente para dar uma resposta satisfatória à maioria das pessoas.

As clínicas de família são dotadas de múltiplos serviços: consultas médicas programadas e de urgência, consultas de enfermagem programadas (principalmente grupos vulneráveis, sendo que algumas são inteira e exclusivamente realizadas por enfermeiros, como por exemplo as gravidezes de baixo risco de mulheres saudáveis) e de urgência (que funcionam como uma triagem, contudo podem por si resolver problemas mais simples. O enfermeiro no RJ tem um papel mais proativo e autónomo, face a Portugal. Além de realizarem autonomamente consultas e múltiplos procedimentos (citologias, colocação de dispositivos intrauterinos, ...), ainda estão habilitados para emitirem receitas de fármacos mais comuns (analgésicos e alguns antibióticos). Existem as visitas domiciliárias, que são feitas em conjunto com os agentes de saúde comunitários - estes gerem os agendamentos de cada equipa, fazem o atendimento inicial na clínica, têm um papel fundamental na ligação a alguns utentes em regime domiciliário, nomeadamente dirigem-se a casa das pessoas para entrega de medicação, credenciais para meios complementares de diagnóstico ou outros documentos, colheita de sangue para análises e ainda analisam a parte social e habitacional dos utentes. Cada equipa de família é constituída por um médico, eventualmente residentes de MFC, um enfermeiro e entre dois a três agentes da comunidade. Para além destes profissionais, cada clínica tem ainda apoio de outras especialidades médicas. A clínica onde fiquei alocada, tinha em permanência um fisiatra e duas psiquiatras, e em regime parcial uma pediatra, um dermatologista e uma ginecologista, que também realizam consultas no local a utentes mais complexos referenciados pelas equipas, assim como estão disponiveis para discussão de casos pontuais. Muitos outros profissionais de saúde estão em permanência ou em determinados dias nas clínicas, nomeadamente dentistas ou higienistas orais, fisioterapeutas (estando muitas clínicas equipadas para realização de tratamentos) e psicólogos. Existe um programa - Academia Carioca - implementado em todas as unidades de saúde no RJ, que têm um espaço destinado à prática de atividade física, com aparelhos de exercício físico, onde se realizam, várias vezes por semana, diferentes atividades (ginástica, dança, exercícios de reforço muscular), acompanhadas por profissionais de desporto. Existe ainda uma farmácia em cada clínica, com um ou dois farmacêuticos presentes, onde são dispensados de forma totalmente gratuita os medicamentos prescritos, que constam numa listagem oficial pré-definida No Brasil, esses tratamentos farmacológicos, apesar de restritos, são totalmente gratuitos e suportados pelo SUS. Excecionalmente, são prescritos outros medicamentos que podem ser adquiridos nas farmácias comunitárias a um custo reduzido. Para além das clínicas de família, a atenção primária é ainda composta pelas Unidade Permanentes de Atendimento (UPA) que são o equivalente aos Serviços de Atendimentos Permanente (SAP) em Portugal. Estas funcionam 24 horas em alguns locais. São unidades equipadas com meios complementares de diagnóstico, nomeadamente análises clínicas, exames radiológicos, eletrocardiografia e ecografia, funcionando assim como "mini" hospitais. Não tive oportunidade de conhecer mais a fundo estas unidades, contudo pelo que me foi transmitido, são locais com grande afluência, onde por vezes os cuidados prestados não são os melhores.

A articulação com os cuidados de saúde secundários dá-se a dois níveis, como em Portugal. Um prende-se com a articulação com o serviço de urgência hospitalar. Existem vagas nos hospitais destinadas a receber exclusivamente doentes referenciados pelas clínicas de família - as denominadas "Vagas Zero". Ou seja, cada serviço de urgência tem camas "reservadas" a doentes urgentes encaminhados pelas clínicas. Antes de se referenciar um doente, o médico envia um pedido informático com a informação clínica para o SAMU (Serviço de 
Atendimento Móvel de Urgência) que dá a orientação de qual o hospital disponível para receber o doente, sendo posteriormente enviada uma ambulância para transporte do mesmo. É mostrado no sistema informático, em tempo real, o estado do pedido, pelo que o médico vai controlando o tempo de chegada do transporte. Do que presenciei, é um serviço com um conceito muito interessante. Contudo, dependendo da gravidade das situações referenciadas, a resposta pode ser algo morosa, dado que o transporte tem de ser feito obrigatoriamente por ambulância. Cheguei a presenciar, não raras vezes, o médico referenciar um doente de manhã/início da tarde e ter de aguardar pela ambulância após a hora de fecho da clínica, várias horas depois.

Outro nível de articulação, são as referenciações para consulta de especialidades hospitalares, que são feitas através de dois sistemas: SISREG e SER.

O SISREG é um sistema municipal exclusivo do Rio de Janeiro e serve para solicitar consultas hospitalares ou procedimentos específicos. Os usuários/utentes que forem referenciados pelo seu médico de família, podem consultar online o estado do pedido, assim como o agendamento de eventuais exames. Foi uma forma de aumentar a transparência dos tempos de espera e permitir que sejam as próprias pessoas a acompanhar o estado do pedido. É um sistema bem concebido do ponto de vista do acesso à informação clínica, sendo que o médico apenas tem de descrever o motivo de referenciação, pois todo o histórico de saúde do utente já está inserido na rede. Existe outro programa, o SER (Sistema Estadual de Regulação), por onde se fazem referenciações para o INCA (Instituto Nacional do Câncer), cirurgia e algumas especialidades hospitalares como hematologia e cardiologia, que é muito mais limitador e burocrático, com tempos de espera muito superiores.

\section{A Prática Clínica Diária}

Após a reunião na coordenação da residência em MFC, dirigi-me à clínica onde fiquei alocada: o Centro Municipal de Saúde Manoel José Ferreira, no Bairro do Catete - RJ. 0 meu tutor era especialista em MFC há 4 anos. Oriundo da Alemanha, fez todo o seu percurso académico no país de origem, onde se formou em Ortopedia, tendo ainda exercido alguns anos. Após um estágio no Brasil, e por se ter deixado fascinar pela MFC, mudou de vida e ingressou na residência em MFC no RJ.

Ao chegar à clínica, após percorrer uma rua relativamente deserta, abri as portas e encontrei o caos. Filas desordeiramente intermináveis, funcionários do aten- dimento embrenhados no trabalho apesar do ruído de fundo, pessoas de bata branca a passarem apressadamente. Pensei que as segundas-feiras são complicadas em todo o lado, contudo vim a aperceber-me que o afluxo e o caos são transversais a todos os dias. Após ter conseguido chamar a atenção de um dos funcionários do atendimento, fui levada ao gabinete do meu tutor, que estava atarefado a meio de uma consulta. Foi uma manhã caótica, com uma agenda muito preenchida, sendo que o meu tutor ia aproveitando os curtos intervalos entre consultas para me ir dando algumas informações. No final da manhã, decorreu uma das reuniões de residentes e preceptores. Curiosamente e como já mencionei, a reunião começou com uma apresentação sobre a experiência de estágio de uma das residentes em Portugal, na USF Oriente, do ACeS Lisboa Oriental. Foi muito interessante escutar a perspetiva dela dos nossos cuidados de saúde, e perceber algumas diferenças apontadas pela mesma, tendo sido tecidos vários elogios ao nosso sistema. Após a hora de almoço, foi mais uma tarde caótica. Dezenas de pessoas inscritas, dezenas de pessoas atendidas, uns casos mais simples, outros mais complexos. Algumas ficaram por atender e foram proteladas para outras alturas (quando o afluxo é grande é feita uma triagem que irei abordar mais à frente). Terminámos já depois das 18h, hora de encerramento da clínica.

Regressei no dia seguinte, às $8 \mathrm{~h}$, hora de abertura da clínica. A clínica funciona todos os dias da semana das $8 \mathrm{~h}$ às $18 \mathrm{~h}$ e aos sábados das $8 \mathrm{~h}$ às $13 \mathrm{~h}$ (apenas para atendimentos urgentes).

Por dia, cada equipa de saúde atende entre 20 a 40 pessoas, por vezes mais, seja em consultas programadas, seja consultas de urgência. Parte dos doentes são vistos pelo residente, em menor número pelo preceptor, pela enfermeira ou por estagiários de medicina, sendo que eu própria realizei várias consultas em autonomia parcial, após ter ganho mais conhecimento do sistema informático. A maioria das consultas programadas, são solicitadas pelos próprios utentes, para resolução de diversos problemas. Nos últimos tempos, por questões políticas e sociais, as pessoas têm perdido os seus seguros de saúde, pelo que passaram a recorrer ao SUS. Foi curioso observar que uma grande parte destes, cidadãos de classe média em idade ativa, agendavam consulta para solicitar "exames de rotina", porque "era isso que faziam todos os anos pelo seguro". À semelhança de Portugal, embora atualmente julgo que estamos a melhorar, ainda há muito a cultura das "rotinas/check ups", sendo que o meu tutor nestes casos tinha uma postura muito correta e tentava educar a população para desmistificar esta necessidade, 
estando também ele alerta para a importância da prevenção quaternária nos cuidados de saúde. De uma forma geral, uns mais relutantes que outros, acabavam por perceber e aceitar.

Existem alguns programas de vigilância, em que as consultas são agendadas periodicamente, como as grávidas e as crianças. À semelhança de Portugal, existem programas para estes grupos, não tão bem estruturados, mas que permitem vigiar algumas das etapas chave do desenvolvimento infantil e da evolução de uma gravidez. Grande parte deste tipo de consulta é feito única e exclusivamente pela enfermeira. Também existem consultas para os hipertensos e para os diabéticos, não havendo critérios bem definidos para a vigilância periódica e realização de MCD's de controlo. Uma das maiores limitações, seja neste tipo de consultas, seja noutros casos, é o tipo de fármacos que podem ser prescritos. O SUS disponibiliza um leque de medicamentos, que são prescritos pelo médico de família e que as pessoas podem levantar gratuitamente na clínica. Contudo não há muitas opções, muito menos se se tratar de fármacos da mesma classe, pelo que se torna muito limitador em caso de efeitos adversos a um determinado fármaco ou mesmo numa escalada terapêutica. Há sempre a opção de os medicamentos serem adquiridos pelas pessoas nas farmácias comunitárias, mas nem toda a gente consegue suportar os custos.

Não há períodos destinados aos vários tipos de consulta num horário semanal, sendo que estas são agendadas consoante a necessidade. Existem alguns períodos em que preferencialmente é agendado um determinado tipo de consulta, mas depende muito da procura, pelo que a maioria das consultas acaba por ser agendada no próprio dia, seja qual for o motivo. É feita uma triagem pelos agentes da comunidade na admissão e estes estão permanentemente a questionar o médico se podem agendar mais alguém. Aliás, as interrupções na consulta são uma constante, seja pelos agentes da comunidade, seja por outros profissionais, ou até pelos próprios doentes, que abrem a porta para questionar se ainda vão ter de esperar muito ou, não raras vezes, para expor logo o problema que os faz estar a procurar ajuda. É muito difícil gerir todo este movimento. Por vezes, não há mesmo capacidade para observar mais pessoas, pelo que os médicos de família, após terem recebido uma solicitação pelos agentes da comunidade e não se tratando de casos urgentes, protelam a consulta para o dia seguinte.

Voltando aos motivos de consulta, os mais comuns são muito variados: uma parte vinha "por rotina, fazer check-ups”, outros para controlo de doenças crónicas (HTA,
DM); As queixas músculo-esqueléticas e os quadros infeciosos são comuns; Outros vinham para as consultas de vigilância de saúde infantil ou saúde materna. Em suma, muitos dos principais motivos são semelhantes ao nosso país. o que vi de diferente: as agressões a desconhecidos, as vítimas das mais variadas situações de violência, a droga e a toxicodependência.

\section{Um exemplo}

Recordo-me de uma consulta, em que um pai trouxe o filho de um ano a uma consulta por um quadro respiratório sugestivo de nasofaringite viral. A consulta durou quase uma hora. Eram os últimos do dia. O meu tutor conhecia a família. Residiam na favela perto da clínica - o Morro Azul. o pai era um conhecido traficante de droga. Abertamente, disse-nos que recentemente tinha criado uma estufa de cogumelos "mágicos" (alucinogénios) em casa, onde reside com o filho e outra criança mais velha da sua atual companheira. Foi interessante observar a forma como o meu tutor foi cautelosamente fazendo algumas questões para averiguar o risco a que a criança estava exposta. Após nos ter ensinado todo o processo e cuidados de cultivo, ficámos a saber que o faria numa área da casa que estaria fechada à chave, pelo que as crianças não teriam acesso. Achei toda a situação muito preocupante, porque o pai também não demonstrou qualquer preocupação face aos riscos que as crianças corriam. 0 meu tutor disse-me que os sistemas de proteção e as autoridades já estavam alerta, mas que de facto nada era feito. Não me vou estender sobre a já mediática violência no Brasil e felizmente não a experienciei, mas vi testemunhos dela, demasiados. Como interna de MGF numa USF modelo B, numa zona urbana, com uma população variada, mas em geral com um nível de socioeconómico médio-alto, nunca me tinha deparado frente a frente com muitas destas situações. Foi muito enriquecedor. Permitiu-me refletir sobre as assimetrias sociais e como é difícil ser médico de família nestes contextos.

O dia-a-dia na clínica também me permitiu colaborar e observar vários procedimentos, desde realização de ECG a suspeitas de EAM, às coloquiais colpocitologias, colocação de DIU de cobre (método muito usado para além dos contracetivos orais), tratamento de feridas, úlceras, entre outros. Relato ainda a minha experiência em participar numa das múltiplas atividades que estavam disponíveis na clínica - a Cuca Fresca. Trata-se de um grupo de autoajuda orientado por um psiquiatra e duas psicólogas que trabalham diariamente na clínica. Este grupo reúne-se uma vez por semana e é constituído por cerca de 30 pessoas, embora o número de 
participantes seja livre. É a equipa de saúde que propõe à pessoa com psicopatologia para procurar e participar neste grupo. Não é necessária inscrição, basta aparecer. Fui assistir a uma dessas sessões. A maioria dos participantes já se conheciam, e eram sobretudo mulheres. Havia duas pessoas novas e eu. Iniciámos a sessão com um quebra-gelo: dissemos o nosso nome e completámos a frase: "hoje eu agradeço...", e em seguida cantámos em grupo. Depois seguiu-se uma sessão em que quem quisesse expunha um problema, um assunto sobre o qual quisesse falar. Foi muito interessante escutar tantas histórias. Pontualmente as psicólogas ou as psiquiatras iam sabiamente intervindo, assim como outros participantes. Terminámos a sessão em círculo, de mãos dadas, a completar a frase: "hoje é um bom dia, porque....". Palpava-se a leveza e a serenidade com que as pessoas saíram da sessão. Mesmo o que nos relatavam na consulta, era um feedback muito positivo. Em Portugal há uma grande carência na área de saúde mental e, neste aspeto, assim como na área do exercício físico e dos cuidados dentários, foi muito gratificante ver como o RJ conseguiu implementar tão importantes áreas no seu sistema de saúde.

Falo, por último, das visitas domiciliárias. Tive oportunidade de realizar várias visitas domiciliárias durante o estágio. Relato uma das mais peculiares. Foi uma visita conjunta com o meu tutor, a enfermeira, o agente da comunidade (que acompanham sempre o médico ou o enfermeiro) e uma das psiquiatras da clínica. Tratavase de uma senhora de 70 anos, cujo domicílio tinha sido solicitado por um vizinho, por uma alteração do comportamento nos últimos tempos. Foi o porteiro do prédio que nos levou até casa da senhora. A situação da casa era indescritível. O cheiro, a acumulação de objetos, a escuridão. Surgiu-nos uma senhora visivelmente emagrecida, com uma higiene precária, com ar desconfiado e assustado com a nossa presença. Feita uma abordagem inicial, em que nos apresentámos e explicámos a nossa presença, seguiu-se uma longa e surreal conversa, principalmente com o meu tutor e a psiquiatra. Era uma senhora viúva, cujo filho tinha também falecido há alguns anos, e que nos últimos meses tinha começado a ter um comportamento estranho, isolando-se em casa e com um discurso incoerente. Apuraram-se muitas ideias persecutórias, entre outros sintomas psicopatológicos. Foi muito enriquecedor observar a forma como a psiquiatra abordou a senhora, tendo tentado negociar uma ida à clínica para um exame físico geral e/ou início de alguma medicação. Não se conseguiu colaboração por parte da senhora, que não apresentava qualquer crítica para a sua situação. Posteriormente, fomos falar com o porteiro, que era a única pessoa que a senhora deixava entrar no apartamento e quem lhe levava algumas refeições. Apurou-se que haveria um sobrinho, que seria a única família da senhora, pelo que o plano seria contactá-lo na tentativa de se conseguir prestar ajuda à mesma. Este não é um caso isolado. Presenciei vários casos de desamparo e pobreza ao longo da minha visita no RJ, não só nas visitas que realizei na favela Morro Azul (pertencente à área da Clínica onde estagiei). Deslocávamo-nos sempre a pé, nunca senti a tão famosa insegurança do Brasil, mas sem dúvida que senti a miséria e a impotência perante tantas situações, das mais chocantes, foram os "moradores de rua” (sem-abrigo) crianças, com problemas de toxicodependência, tendo presenciado uma ação de uma equipa especial de recolha destas crianças para instituições de onde, sistematicamente, fugiam novamente para a rua...

\section{Conclusões}

Haveria mais para contar das duas semanas que passei no RJ. Foi uma experiência única. Permitiu-me conhecer novas realidades, ter novas perspetivas. Impressionou-me a organização, o rápido e exemplar desenvolvimento e formação dos cuidados de saúde primários do RJ. Impressionou-me a proatividade, o desempenho e a dedicação dos residentes de MFC. Foi interessante lidar com tantos problemas, uns comuns e semelhantes aos portugueses, outros com que nunca me tinha deparado, mas com que me poderei deparar no imprevisível futuro. Aprendi muito com o meu tutor, sendo que o facto dele próprio já ter exercido na Europa, permite outro olhar sobre o Brasil. Aprendi com todos os profissionais com que me cruzei e com todos aqueles que precisavam da nossa ajuda. Foi um estágio que me permitiu conhecer um sistema de saúde diferente, jovem, com muito potencial para progredir e melhorar os resultados em saúde do RJ. Pude também conhecer o programa de formação em MFC, do qual saliento a organização e o equilíbrio entre o treino da consulta e a gestão da prática clínica, não descurando a parte da atualização científica e partilha de informação interpares. 0 dia a dia na clínica foi preenchido, com muitas horas de trabalho. Apesar de em Portugal também trabalharmos várias horas extra, o ritmo na clínica onde estive era alucinante. Isso permitiu-me conhecer a cultura de saúde, num sítio onde, por vezes, a pobreza, a violência, as condições de higiene precárias e as condições sociais de pobreza têm um papel predominante na condição de saúde individual. Sem dúvida, foram duas semanas muito enriquecedoras em termos profissionais, mas também pessoais. Cruzei-me com muitas histórias e pude explorar um pouco a cidade carioca, que bem me acolheu e cuja beleza superou largamente as minhas 
expectativas. Para além do enriquecimento e crescimento pessoal, conto usar toda esta experiência para prestar melhores serviços e cuidados como futura médica de família.

\section{Agradecimentos}

Dr. Michael Thomae (tutor do estágio) Inaiara Bragante (secretária do PRMFC)

Toda a equipa do Centro Municipal de Saúde Manoel José Ferreira (RJ - Brasil)

Dra Marta Marquês (orientadora de formação)

\section{Referências Bibliográficas}

1. World Health Organization and UNICEF. 1978. Report of the International Conference on Primary Health Care. Alma Ata, USSR, 6-12 $2^{\text {th }}$ September 1978

2. Estatísticas Demográficas 2017. Editor: Instituto Nacional de Estatística, IP (C) INE, I.P., Lisboa - Portugal, 2018

3. Relatório de Imigração Fronteiras e Asilo. SEF

4. População estrangeira com estatuto legal de residente: total e por algumas nacionalidades. Fontes de Dados: INE | SEF/MAI - População Estrangeira com Estatuto Legal de Residente. Fonte: PORDATA. Consultado a 22/04/2019

5. Harzheim E. Reforma da atenção primária à saúde na cidade do Rio de Janeiro - avaliação dos primeiros três anos de clínicas da família. 2013. Available at: https:// www.ufrgs.br/telessauders/documentos/biblioteca_em_ saude/050_material_saude_livro_reforma_aps_rj.pdf. Accessed May 16; 2019

6. Matta GC, Morosini MVG. Atenção Primária à Saúde. (Dicionário da Educação Profissional em Saúde web site). 2009. Available at: http://www.sites.epsjv.fiocruz.br/dicionario/ verbetes/ateprisau.html. Accessed May 16; 2019

Conflito de Interesses

Nada a declarar. 